\title{
Genome-wide DNA methylation analysis of pseudohypoparathyroidism patients with GNAS imprinting defects
}

\author{
Anne Rochtus ${ }^{1,2}$, Alejandro Martin-Trujillo ${ }^{3}$, Benedetta Izzi ${ }^{1}$, Francesca Elli ${ }^{4}$, Intza Garin ${ }^{5}$, Agnes Linglart ${ }^{6,7}$, \\ Giovanna Mantovani ${ }^{4}$, Guiomar Perez de Nanclares ${ }^{5}$, Suzanne Thiele ${ }^{8}$, Brigitte Decallonne ${ }^{9}$, Chris Van Geet ${ }^{2}$, \\ David Monk ${ }^{3}$ and Kathleen Freson ${ }^{1 *}$
}

\begin{abstract}
Background: Pseudohypoparathyroidism (PHP) is caused by (epi)genetic defects in the imprinted GNAS cluster. Current classification of PHP patients is hampered by clinical and molecular diagnostic overlaps. The European Consortium for the study of PHP designed a genome-wide methylation study to improve molecular diagnosis.

Methods: The HumanMethylation 450K BeadChip was used to analyze genome-wide methylation in 24 PHP patients with parathyroid hormone resistance and 20 age- and gender-matched controls. Patients were previously diagnosed with GNAS-specific differentially methylated regions (DMRs) and include 6 patients with known STX16 deletion $\left(\mathrm{PHP}^{\Delta \mathrm{st} \times 16}\right)$ and 18 without deletion (PHP ${ }^{\text {neg }}$ ).

Results: The array demonstrated that PHP patients do not show DNA methylation differences at the wholegenome level. Unsupervised clustering of GNAS-specific DMRs divides PHP ${ }^{\Delta s t x 16}$ versus PHP neg patients. Interestingly, in contrast to the notion that all PHP patients share methylation defects in the A/B DMR while only PHP ${ }^{\Delta s \times 16}$ patients have normal NESP, GNAS-AS1 and XL methylation, we found a novel DMR (named GNAS-AS2) in the GNASAS1 region that is significantly different in both $\mathrm{PHP}^{\Delta \mathrm{st} \times 16}$ and $\mathrm{PHP}{ }^{\text {neg }}$, as validated by Sequenom EpiTYPER in a larger PHP cohort. The analysis of 58 DMRs revealed that 8/18 PHPneg and 1/6 PHP ${ }^{\Delta s t \times 16}$ patients have multi-locus methylation defects. Validation was performed for FANCC and SVOPL DMRs.
\end{abstract}

Conclusions: This is the first genome-wide methylation study for PHP patients that confirmed that GNAS is the most significant DMR, and the presence of STX16 deletion divides PHP patients in two groups. Moreover, a novel GNAS-AS2 DMR affects all PHP patients, and PHP patients seem sensitive to multi-locus methylation defects.

Keywords: Pseudohypoparathyroidism, GNAS, DNA methylation, Imprinting disorders

\section{Background}

Genomic imprinting is a parent-of-origin dependent gene expression that is essential for mammalian development. A mechanism underlying allele-specific expression is DNA methylation. The addition of a methyl group to DNA cytosine nucleotides at $\mathrm{CpG}$ sites can occur in an allele-specific manner, and allele-specific methylation in imprint control regions (referred to as differentially

\footnotetext{
* Correspondence: kathleen.freson@med.kuleuven.be

'Department of Cardiovascular Sciences, Center for Molecular and Vascular Biology, University of Leuven, Campus Gasthuisberg, O\&N1, Herestraat 49, Box 911, 3000 Leuven, Belgium

Full list of author information is available at the end of the article
}

methylated regions (DMRs)) is associated with parent-oforigin dependent gene expression. Imprinting disorders are a group of rare diseases affecting growth, development, and metabolism that are associated with (epi)genetic disruption of imprinting genes [1]. Pseudohypoparathyroidism (PHP) is a rare endocrine disorder that can be caused by genetic or epigenetic alterations in the imprinted cluster GNAS localized on chromosome 20q13.3 [2]. The human GNAS locus harbors four DMRs encompassing the promoters of four alternative transcripts: exon A/B (GNAS- $A / B$ : TSS $\mathrm{DMR}=\boldsymbol{A} / \boldsymbol{B})$, GNAS antisense (GNAS-AS1: TSS DMR $=$ $A S 1)$, extra-large stimulatory G protein (GNAS-XL: Ex1 
$\mathrm{DMR}=\boldsymbol{X L}$ ), and neuroendocrine secretory protein 55 (GNAS-NESP: TSS DMR = NESP) (Fig. 1a) [3]. PHP type I (PHP1A and PHP1B) patients are characterized by endorgan resistance to the action of the parathyroid hormone $(\mathrm{PTH})$, which leads to hypocalcemia, hyperphosphatemia, and elevated levels of PTH in the absence of vitamin D deficiency. They often also have thyroid-stimulating hormone (TSH) resistance $[2,3]$. PHP1A patients have in addition to hormone resistance clinical features collectively referred to as Albright's hereditary osteodystrophy (AHO) that include brachydactyly, short stature, and round face; they may also present with obesity, subcutaneous ossifications, mental retardation, and behavior problems $[4,5]$. PHP1A is caused by heterozygous maternally inherited inactivating mutations in the coding sequence of Gs $\alpha$ (exons 1 to 13 of GNAS) [2, 3, 6]. On the other hand, paternally inherited inactivating mutations lead to pseudopseudohypoparathyroidism (PPHP), characterized by AHO features but without hormone resistance $[2,3,6]$. Most patients affected with the PHP1B form of the disease exhibit mainly PTH resistance and subclinical $\mathrm{TSH}$ resistance and do not have $\mathrm{AHO}$ features. In some cases however, typical brachydactyly, severe obesity [7], and congenital hypothyroidism [8,9] have been described in PHP1B patients highlighting the overlap between PHP1A and PHP1B. All PHP1B patients have methylation abnormalities in the GNAS cluster with loss of imprinting at the $A / B$ DMR [6]. The familial form of PHP1B (often referred to as autosomal dominant AD-PHP1B) is typically associated with microdeletions in the STX16 region located upstream of the GNAS cluster [10-13] and less frequently with deletion removing the NESP DMR [14]. On the other hand, sporadic PHP1B patients have broad GNAS methylation defects that involve all four GNAS DMRs without a known underlying genetic cause $[2,3,6]$.

However, from recent data, it becomes clear this original PHP classification is no longer accurate for the following four reasons: (i) broad GNAS methylation defects were also found for PHP1B patients with an AHO phenotype [7, 15-17], (ii) the degree of the methylation defect seems not to correlate with the disease severity [18], (iii) reduced Gs $\alpha$ activity is no longer exclusive for PHP1A with inactivating mutations but recently also was described for PHP patients with epigenetic defects $[19,20]$, and (iv) partial GNAS methylation defects have

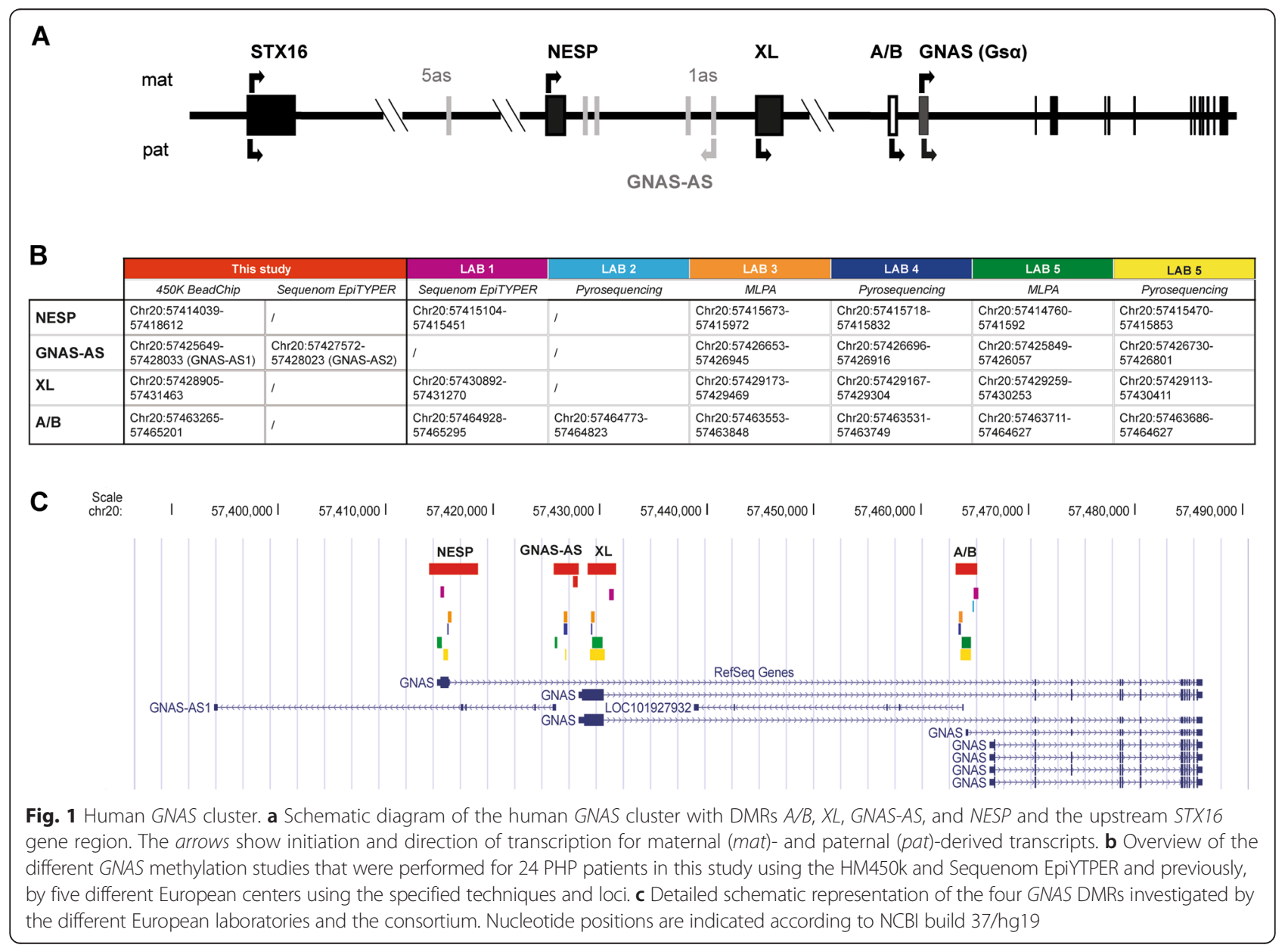


been described [21]. Moreover, as shown for other imprinting disorders, such as Silver-Russell or BeckwithWiedemann syndrome, also some PHP1B patients were shown to have multi-locus imprinting defects as studied by a targeted approach that comprised known imprinted loci [22-24]. Therefore, there is a strong need to improve the current classification of PHP patients that might be feasible based on more detailed (epi)genetic or clinical data using larger patient cohorts analyzed with more powerful techniques. The European Consortium for the study of PHP (EuroPHP) designed the present genome-wide methylation study to gain insight in epigenetic profiles that might improve an epigenetic-based classification. We have analyzed the methylomes of 24 previously diagnosed PHP patients with GNAS epigenetic defects recruited from five European centers. This sample set includes 6 patients with the STX16 3-kb recurrent microdeletion $\left(\mathrm{PHP}^{\Delta \mathrm{stx} 16}\right)[11]$ and 18 without any identified deletion within the GNAS locus $\left(\mathrm{PHP}^{\text {neg }}\right)$. The following analyses were performed: (i) changes in DNA methylation at the genome-wide level, (ii) unsupervised cluster analysis for the GNAS locus and comparisons with previous GNAS methylation data used for PHP diagnosis, and (iii) detailed DNA quantification analysis for other imprinted DMRs. DNA methylation data were validated with the Sequenom EpiTYPER for additional PHP patients with PTH resistance and proven epigenetic defects.

\section{Results}

Genome-wide DNA methylation analysis for PHP patients The Illumina Infinium HumanMethylation 450K BeadChip (HM450k) was used to determine genome-wide DNA methylation profiles in 24 PHP patients and 20 age- and gender-matched healthy controls. Table 1 presents the clinical characteristics of the PHP patients that all have PTH resistance and hypocalcemia. Mild AHO features were only present in patients $8,10,13,15$, and 20. All 24 PHP patients were previously diagnosed with a GNAS epigenetic defect using different methodologies (Fig. 1b, c), and methylation values for the different GNAS DMRs obtained by these assays are shown in Table 1 . We have included 6 PHP patients with a deletion of the STX16 region upstream of GNAS and hypomethylation of the $A / B$ DMR only (referred to as $\mathrm{PHP}^{\Delta \mathrm{stx} 16}$ ) and $18 \mathrm{PHP}$ patients without the deletion and full or partial methylation defects in $A / B, X L, A S 1$, and NESP DMRs (referred to as PHP ${ }^{\text {neg }}$ ) (Table 1). Hierarchical cluster analysis of all CpGs according to the different subtypes $\mathrm{PHP}^{\Delta \text { stx16}}, \mathrm{PHP}^{\text {neg }}$, or control (Fig. 2a) and unsupervised hierarchical clustering analysis of the data (Fig. 2b) showed that the samples are clustered irrespective of the subgroup, suggesting that there are no subgroup differences at whole-genome level. To further exclude a global methylation defect in PHP patients, we also analyzed the methylation of DNA repetitive elements (LINE-1 and LINE-2) that again showed no differences between patients and controls (data not shown).

\section{GNAS methylation analysis based on HM450k data for PHP patients}

The methylation values for all CpGs located within the GNAS cluster were extracted from data obtained from the genome-wide methylation analysis (Additional file 1: Table S1). The HM450k contains probes mapping to four regions that cover the four GNAS DMRs as specified by red boxes in Fig. 1c (referred to as $A / B, X L, A S 1$, and NESP). These regions are larger but overlap with the regions that were previously investigated by five European centers that have used different technologies including MS-MLPA, Sequenom EpiTYPER, or pyrosequencing to quantify the methylation of the GNAS DMRs for the 24 PHP patients (see Fig. 1c for the location of the studied regions and methodologies and Table 1 for the results for each patient). Unsupervised hierarchical cluster analysis of GNAS methylation data obtained from the HM450k for the 24 PHP patients divided these patients in 2 groups according to the presence/absence of STX16 deletion $\left(\mathrm{PHP}^{\Delta \mathrm{stx} 16}\right.$ versus $\mathrm{PHP}^{\text {neg }}$ ) (Fig. 3). As known in literature $[2,3]$ and confirming the previous diagnosis that was made by the 5 laboratories, $\mathrm{PHP}^{\Delta \text { stx16 }}$ only showed hypomethylation of DMR $A / B$ while PHP ${ }^{\text {neg }}$ patients had abnormal methylation for all 4 GNAS DMRs. However, 2 PHP $^{\text {neg }}$ patients (23 and 24) were clustered closer to the healthy control population having normal methylation values for the all GNAS DMRs (Table 1 and Fig. 3). These patients were previously identified with a "partial" GNAS methylation defect using MS-MLPA and pyrosequencing respectively. After analyzing the separate $\mathrm{CpGs}$ from the HM450k, no significant differences were found compared to the control population (separated CpGs are visualized in Fig. 3). At the individual probe level, we noticed that both patients were mosaic and patient 23 was slightly more hypomethylated than patient 24 .

Interestingly, a smaller region we referred to as $A S 2$ within the AS1 amplicon had significantly lower methylation values for both $\mathrm{PHP}^{\Delta \text { stxi6 }}$ and $\mathrm{PHP}^{\text {neg }}$ patients compared to the controls (Fig. 3 and Table 1). This AS2 region does not overlap with amplicons that were previously studied for the $A S$ region by MS-MLPA, Sequenom EpiTYPER, or pyrosequencing by the different laboratories (Fig. 1b, c and Additional file 2: Figure S1). The $A S 2$ region is separated from $X L$ by a hypermethylated region (Fig. 3). To confirm AS2 hypomethylation, a validation study was developed using the Sequenom EpiTYPER to quantify methylation in the AS2 region (Additional file 2: Figure S1) in 42 PHP patients with 
Table 1 Clinical and molecular characteristics of the PHP patients enrolled in the genome-wide DNA methylation study

\begin{tabular}{|c|c|c|c|c|c|c|c|c|c|c|c|c|c|c|c|c|c|c|c|c|}
\hline \multicolumn{9}{|c|}{ 1. Clinical characteristics } & \multicolumn{7}{|c|}{ 2. Initial diagnostic screening } & \multicolumn{5}{|c|}{ 3. HM450k } \\
\hline Patient & Gender & PTH-res & TSH-res & $\mathrm{Ca} \downarrow$ & $P \uparrow$ & OS & BD & Additional features & STX16 & $L A B$ & Label & NESP & AS & $\mathrm{XL}$ & $A / B$ & NESP & AS1 & AS2 & $\mathrm{XL}$ & $A / B$ \\
\hline 1 & $\mathrm{~F}$ & Yes & Yes & Yes & Yes & No & No & $\begin{array}{l}\text { Two café-au-lait spots, autoimmune } \\
\text { thyroiditis }\end{array}$ & & 4 & Full PHP1B_broad & 94 & 7 & 27 & 3 & $90^{* *}$ & $10^{* *}$ & $8^{* *}$ & $34^{*}$ & $13^{* *}$ \\
\hline $2^{*}$ & M & Yes & Yes & Yes & Yes & No & No & & & 3 & Full PHP1B—broad & 80 & 7 & 8 & 2 & $89^{* *}$ & $10^{* *}$ & $8^{* *}$ & $6^{* *}$ & $12^{* *}$ \\
\hline 3 & M & Yes & & Yes & Yes & No & No & Von Willebrand disease & & 5 & Full PHP1B_broad & 88 & 4 & 6 & 0 & $88^{* *}$ & $10^{* *}$ & $8^{* *}$ & $7^{* *}$ & $13^{* *}$ \\
\hline $4^{*}$ & M & Yes & No & Yes & Yes & No & No & & & 1 & Full PHP1B_broad & 92 & & 8 & 7 & $89^{* *}$ & $10^{* *}$ & $8^{* *}$ & $7^{* *}$ & $13^{* *}$ \\
\hline $5^{*}$ & M & Yes & No & Yes & Yes & No & No & & & 3 & Full PHP1B—broad & 82 & 7 & 8 & 2 & $89^{* *}$ & $9^{* *}$ & $8^{* *}$ & $7^{* *}$ & $12^{* *}$ \\
\hline 6 & $\mathrm{~F}$ & Yes & No & Yes & Yes & No & No & & & 1 & Full PHP1B—broad & 92 & & 10 & 9 & $89^{* *}$ & $9^{* *}$ & $7^{* *}$ & $5^{* *}$ & $12^{* *}$ \\
\hline 7 & M & Yes & No & Yes & Yes & No & No & $\begin{array}{l}\text { Bilateral cryptorchidism, osteopenia } \\
\text { of hands, behavior problems }\end{array}$ & & 4 & Full PHP1B_broad & 95 & 5 & 4 & 3 & $89^{* *}$ & $9^{* *}$ & $8^{* *}$ & $5^{* *}$ & $12^{* *}$ \\
\hline $8^{*}$ & $\mathrm{~F}$ & Yes & & Yes & No & No & Yes & & & 5 & Full PHP1B—broad & 90 & 3 & 4 & 0 & $89^{* *}$ & $10^{* *}$ & $8^{* *}$ & $6^{* *}$ & $12^{* *}$ \\
\hline 9 & $\mathrm{~F}$ & Yes & Yes & Yes & No & & No & Enchondromatosis & & 5 & Partial PHP1B_-broad & 83 & 23 & 34 & 33 & 63 & 27 & 23 & $24^{*}$ & 35 \\
\hline 10 & $\mathrm{~F}$ & Yes & No & Yes & Yes & No & Yes & Exostosis & & 4 & Partial PHP1B_-broad & 84 & 20 & 24 & 13 & $75^{*}$ & $22^{*}$ & $16^{* *}$ & $33^{*}$ & 31 \\
\hline 11 & $\mathrm{~F}$ & Yes & & & & & & & & 5 & Partial PHP1B—broad & 80 & 10 & 27 & 14 & $78^{*}$ & $23^{*}$ & $19^{*}$ & $34^{*}$ & 28 \\
\hline $12^{*}$ & M & Yes & Yes & Yes & No & No & No & $\begin{array}{l}\text { Severe hypertension with organ damage, } \\
\text { hypercalciuria }\end{array}$ & & 3 & Full PHP1B_broad & 77 & 8 & 8 & 3 & $75^{*}$ & $12^{* *}$ & $8^{* *}$ & $7^{* *}$ & $17^{* *}$ \\
\hline $13^{*}$ & $\mathrm{~F}$ & Yes & Yes & Yes & Yes & No & Yes & & & 3 & Partial PHP1A—broad & 76 & 8 & 7 & 5 & $73^{*}$ & $15^{* *}$ & $9^{* *}$ & $5^{* *}$ & $21^{*}$ \\
\hline $14^{*}$ & M & Yes & Yes & Yes & No & No & No & & & 3 & Partial PHP1B—broad & 80 & 10 & 19 & 29 & $76^{*}$ & $19^{* *}$ & $13^{* *}$ & $30^{*}$ & $21^{*}$ \\
\hline 15 & $\mathrm{~F}$ & Yes & Yes & Yes & Yes & Yes & No & $\begin{array}{l}\text { Langerhans cell histiocytosis, neonatal } \\
\text { hydrocephaly, sessile exostosis }\end{array}$ & & 4 & Partial PHP1A—broad & 86 & 18 & 18 & 7 & $81^{* *}$ & $18^{* *}$ & $15^{* *}$ & $32^{*}$ & $21^{*}$ \\
\hline 16 & $\mathrm{~F}$ & Yes & Yes & Yes & Yes & No & No & $\begin{array}{l}\text { Fahr's syndrome (calcifications of basal } \\
\text { ganglia), autoimmune thyroiditis }\end{array}$ & & 4 & Partial PHP1B_broad & 91 & 12 & 30 & 7 & $85^{* *}$ & $17^{* *}$ & $16^{* *}$ & 43 & $21^{*}$ \\
\hline 17 & $\mathrm{~F}$ & Yes & No & Yes & No & No & No & & $\Delta \mathrm{STX} 16$ & 3 & PHP1B_A/B only & 42 & 36 & 44 & 6 & 46 & 36 & $16^{* *}$ & 45 & $14^{* *}$ \\
\hline $18^{*}$ & $\mathrm{~F}$ & Yes & Yes & No & No & & & & $\Delta \mathrm{STX} 16$ & 2 & PHP1B_A/B only & - & - & - & 4 & 50 & 37 & $14^{* *}$ & 47 & $14^{* *}$ \\
\hline 19 & M & Yes & Yes & Yes & Yes & Yes & No & Calcifications of basal ganglia & $\Delta \mathrm{STX} 16$ & 4 & PHP1B_A/B only & 49 & 42 & 52 & 3 & 50 & 41 & $18^{* *}$ & 48 & $15^{* *}$ \\
\hline 20 & $\mathrm{~F}$ & Yes & No & Yes & Yes & No & Yes & & $\Delta \mathrm{STX} 16$ & 1 & PHP1B_A/B only & 39 & - & 36 & 10 & 50 & 40 & $16^{* *}$ & 50 & $15^{* *}$ \\
\hline 21 & M & Yes & Yes & No & Yes & & & & $\Delta \mathrm{STX} 16$ & 2 & PHP1B_A/B only & - & - & & 4 & 52 & 41 & $16^{* *}$ & 51 & $13^{* *}$ \\
\hline 22 & M & Yes & No & Yes & No & No & No & & $\Delta \mathrm{STX} 16$ & 1 & PHP1B_A/B only & 29 & - & 38 & 14 & 53 & 42 & $14^{* *}$ & 51 & $14^{* *}$ \\
\hline $23^{*}$ & $\mathrm{~F}$ & Yes & No & Yes & Yes & No & No & & & 3 & Partial PHP1B_-broad & 54 & 26 & 30 & 7 & 55 & 36 & 26 & 40 & 41 \\
\hline 24 & M & Yes & No & Yes & Yes & No & No & $\begin{array}{l}\text { Severe osteopenia without } \\
\text { ossifications }\end{array}$ & & 5 & Partial PHP1B_broad & 70 & 35 & 46 & 44 & 57 & 39 & 34 & 41 & 43 \\
\hline
\end{tabular}

From left to right: (1) clinical characteristics; (2) mean methylation values of GNAS studies that were performed previously by five different European centers as described in Fig. 1: and (3) mean methylation values of GNAS investigated with HM450k. Ordering of the patients according to unsupervised hierarchical clustering of GNAS methylation investigated with HM450k. Patients* have methylation changes at imprinted genes other than GNAS. Methylation values are presented as percentage. Mean \pm SD of the 20 controls from the HM450k array is shown below the HM450k columns

$B D$ brachydactyly, $C a$ calcium, $F$ female, LAB laboratory of initial diagnostic screening, annotation as shown in Fig. 1, M male, OS heterotopic ossifications, $P$ phosphate, PTH-res parathyroid hormone resistance, TSH-res thyroid-stimulating hormone resistance, $\triangle S T X 16$ patients with underlying STX16 deletion

${ }^{*}$ Methylation < or $>3 \mathrm{SD}$ outside the mean of the controls

Methylation $<$ or $>3$ SD outside the mean of the controls and $<0.20$ or $>0.80$ absolute methylation 


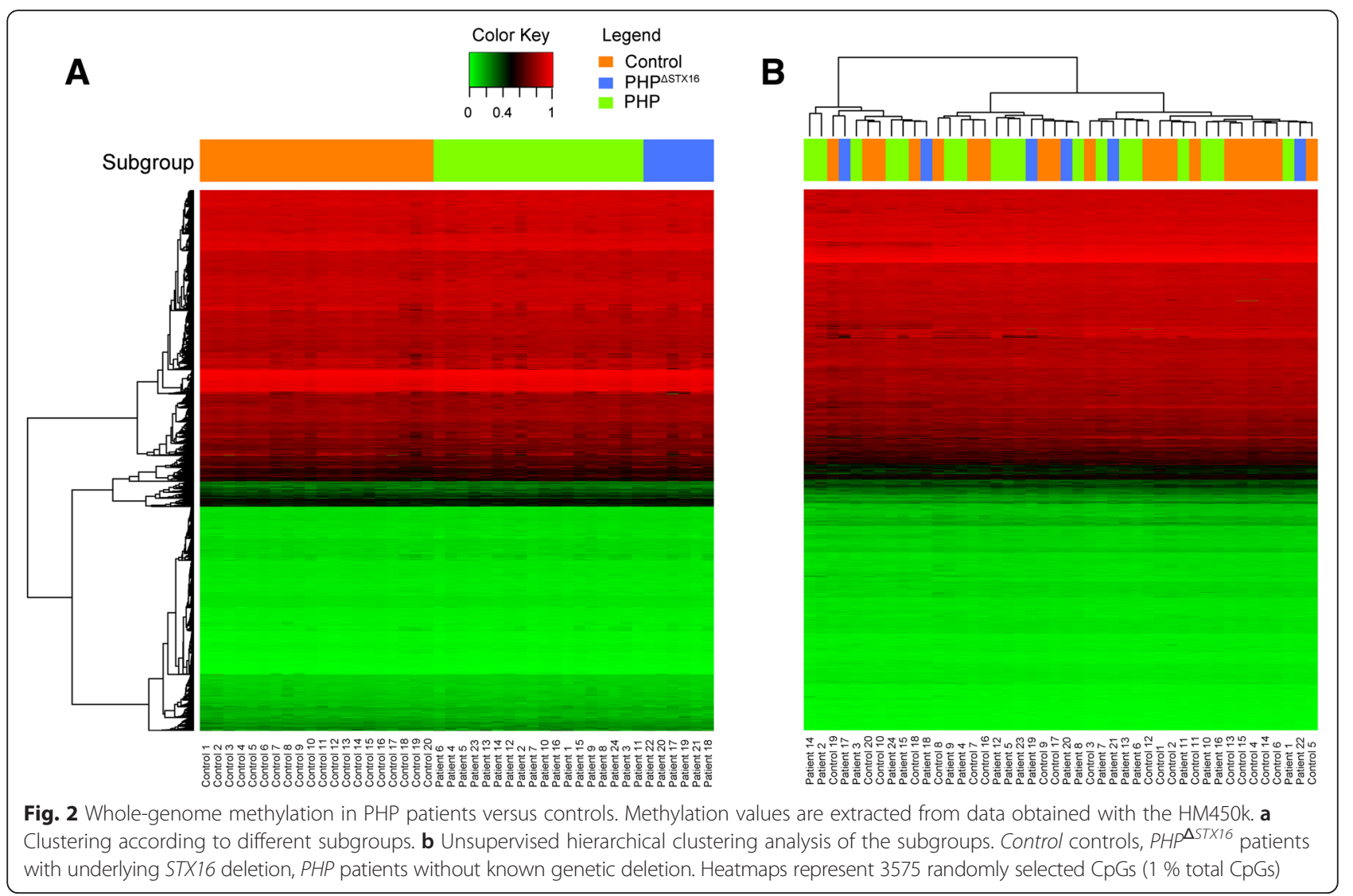

PTH resistance that were previously diagnosed with a GNAS epimutation and 20 age- and gender-matched healthy controls. These $42 \mathrm{PHP}$ patients include 20 $\mathrm{PHP}^{\Delta \mathrm{stx} 16}$ and $22 \mathrm{PHP}^{\text {neg }}$ patients, of which 3 patients in each group were also analyzed by HM450k array (Additional file 1: Table S2). The $22 \mathrm{PHP}^{\text {neg }}$ comprise 20 PHP1B with full or partial broad GNAS methylation defect and 2 PHP1B patients with isolated A/B methylation defect but having no STX16 deletion (Additional file 1: Table S3). Significant AS2 hypomethylation ( $P$ value $<0.0001)$ was detected for both $\mathrm{PHP}^{\Delta \operatorname{stx} 16}$ and $\mathrm{PHP}^{\text {neg }}$ patient groups compared to controls (Fig. 4 and Additional file 1: Table S3). No significant difference in AS2 methylation was found between $\mathrm{PHP}^{\Delta \mathrm{stx} 16}$ and $\mathrm{PHP}^{\text {neg }}$ patients with mean methylation values of $10 \%$ (95\% confidence interval (CI) 7-13\%) versus $8 \%$ (95\% CI 5-11\%), respectively. This suggests that the imprinting defect for this interval is not determined by the STX16 deletion and is specific for PHP1B. To investigate if $A S 2$ hypomethylation changed $X L$ expression, we performed GNAS versus $X L$ messenger RNA (mRNA) expression studies using total blood mRNA from a $\mathrm{PHP}^{\text {neg }}$ patient and control heterozygous for GNAS SNP (rs7121), but we could not detect XL expression in blood cells.

\section{Methylation studies of other imprinted genes for PHP patients}

As multi-locus abnormalities have also been described for PHP1B patients [23], we analyzed the methylation values for all CpGs located within 58 imprinted DMRs for humans that are covered by the HM450k (Additional file 1: Table S4 and S5). In addition to methylation abnormalities for the 4 GNAS DMRs, we found multilocus methylation defects in $8 \mathrm{PHP}^{\text {neg }}$ and $1 \mathrm{PHP}^{\Delta \mathrm{stx} 16}$ patients (Fig. 5). Abnormal methylation was found for 19 of the 58 human imprinted DMRs, and this included significant hypomethylation for PPIEL:Ex1-DMR, DIRAS3:Ex2-DMR, DIRAS3:TSS-DMR, JAKMIP1, NAP1L5:TSSDMR, FAM50B:TSS-DMR, SVOPL, FANCC:Int1-DMR, SNRPN:alt-TSS-DMR, IGF1R:Int2-DMR, LOC100130522/ PARD6G-AS1, WRB:alt-TSS-DMR, and NHP2L1:alt-TSSDMR and significant hypermethylation for ZBDF2/ GPR1:IG-DMR, PEG13:TSS-DMR, RB1:Int2-DMR, SNRPN intragenic CpG40, SNRPN_2, and SNRPN_3. ZBDF2/ GPR1:IG-DMR hypermethylation is likely due to GPR1AS:TSS-DMR hypomethylation. The GPR1-AS:TSS-DMR is not included in our screening, as it acquires immediate biparental methylation following implantation; therefore, we can only infer loss of methylation due to hypermethylation of ZBDF2 [25]. The clinical phenotype of the 


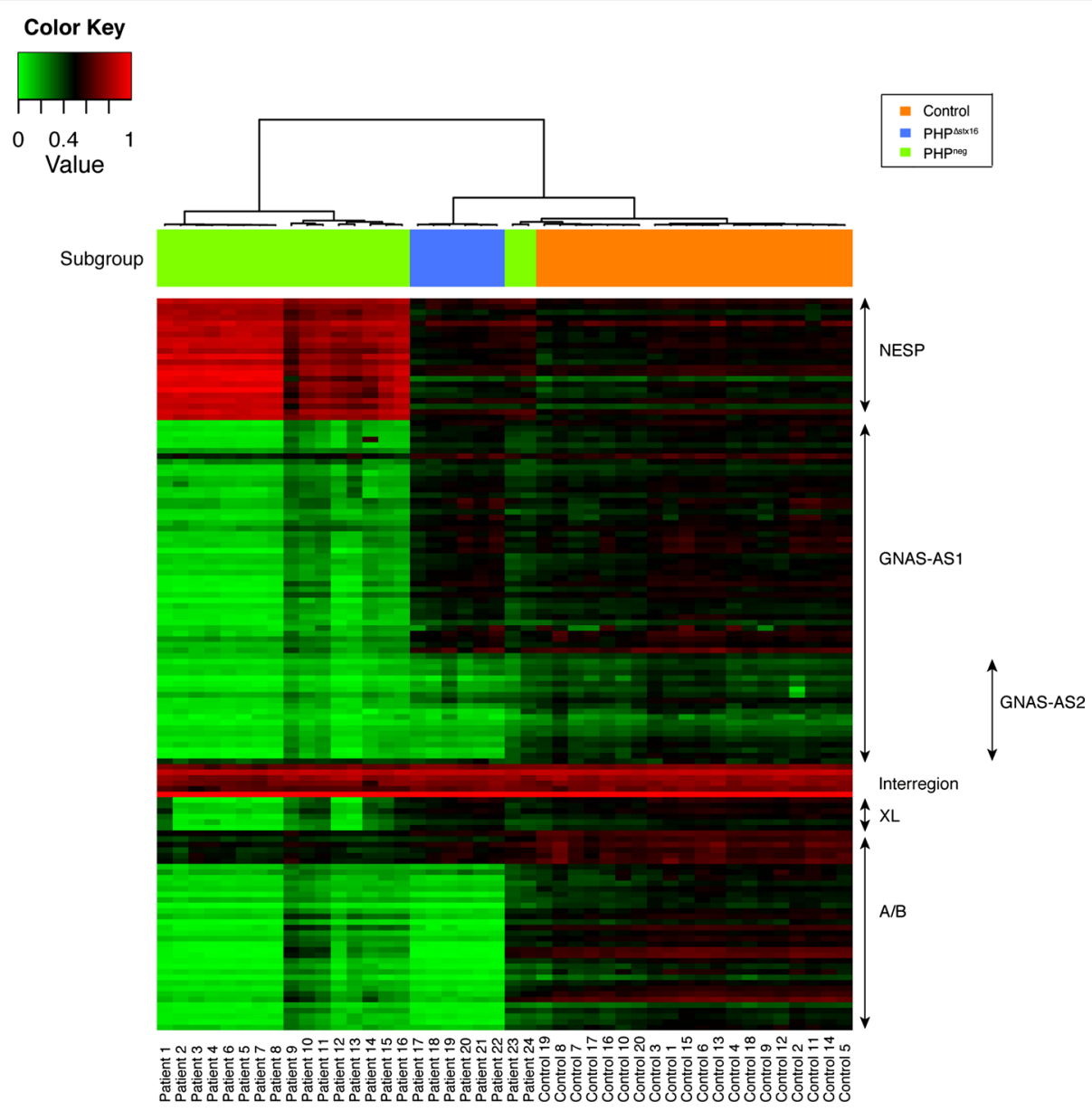

Fig. 3 Unsupervised hierarchical clustering of GNAS methylation in PHP patients. Methylation values of the individual CpGs of the four GNAS DMRs are extracted from data obtained with the HM450k. Green and red represent 0 and 1 methylation, respectively. The arrows show the different GNAS transcripts

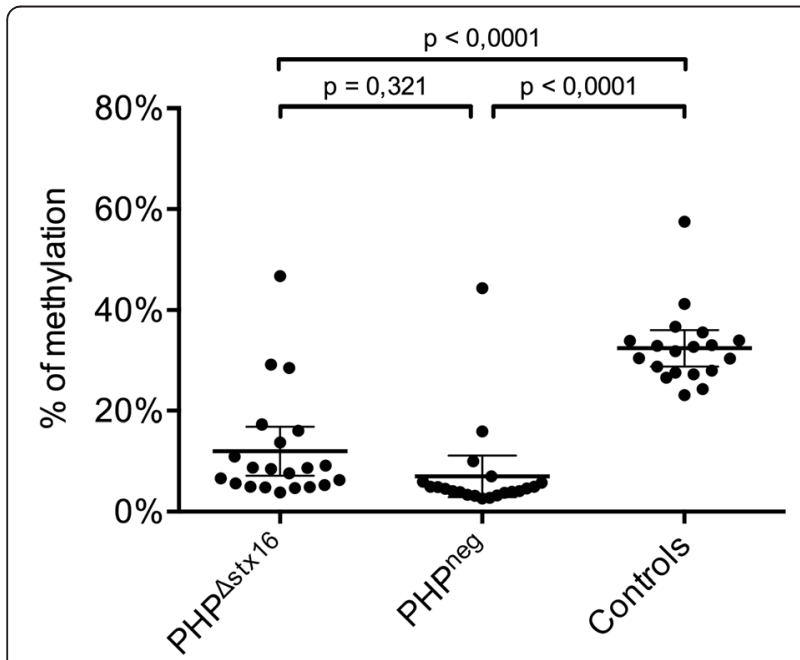

Fig. 4 Scatter plot of NESP-AS2 methylation in PHP patients and controls. Methylation values are obtained with the Sequenom EpiTYPER. Horizontal bars indicate the mean and SD of the group. PHP patients are separated according to underlying STX16 deletion patients with multi-locus methylation abnormalities was not different from the PHP patients with GNASspecific epimutations (Table 1).

To confirm the findings of multi-locus abnormalities, we performed a validation study using the Sequenom EpiTYPER for 26 PHP patients and 12 healthy controls. We have selected FANCC and SVOPL as hypomethylation was seen in 3 and 7 PHP patients, respectively. We also validated the methylation of WDR27 amplicon as negative control. The 26 PHP patients included 5 $\mathrm{PHP}^{\Delta \text { stx16 }}$ and $21 \mathrm{PHP}^{\text {neg }}$ patients; 3 patients of each group were also analyzed by HM450k (Additional file 1: Table S2). Eight patients had multi-locus methylation abnormalities (Fig. 6 and Additional file 1: Table S6). The most significant methylation difference was found for SVOPL in three PHP ${ }^{\text {neg }}$ patients.

\section{Discussion}

Genome-wide studies to identify genetic alterations have successfully entered the field of rare diseases [26]. Similar attempts to elucidate the epigenome are expected to 


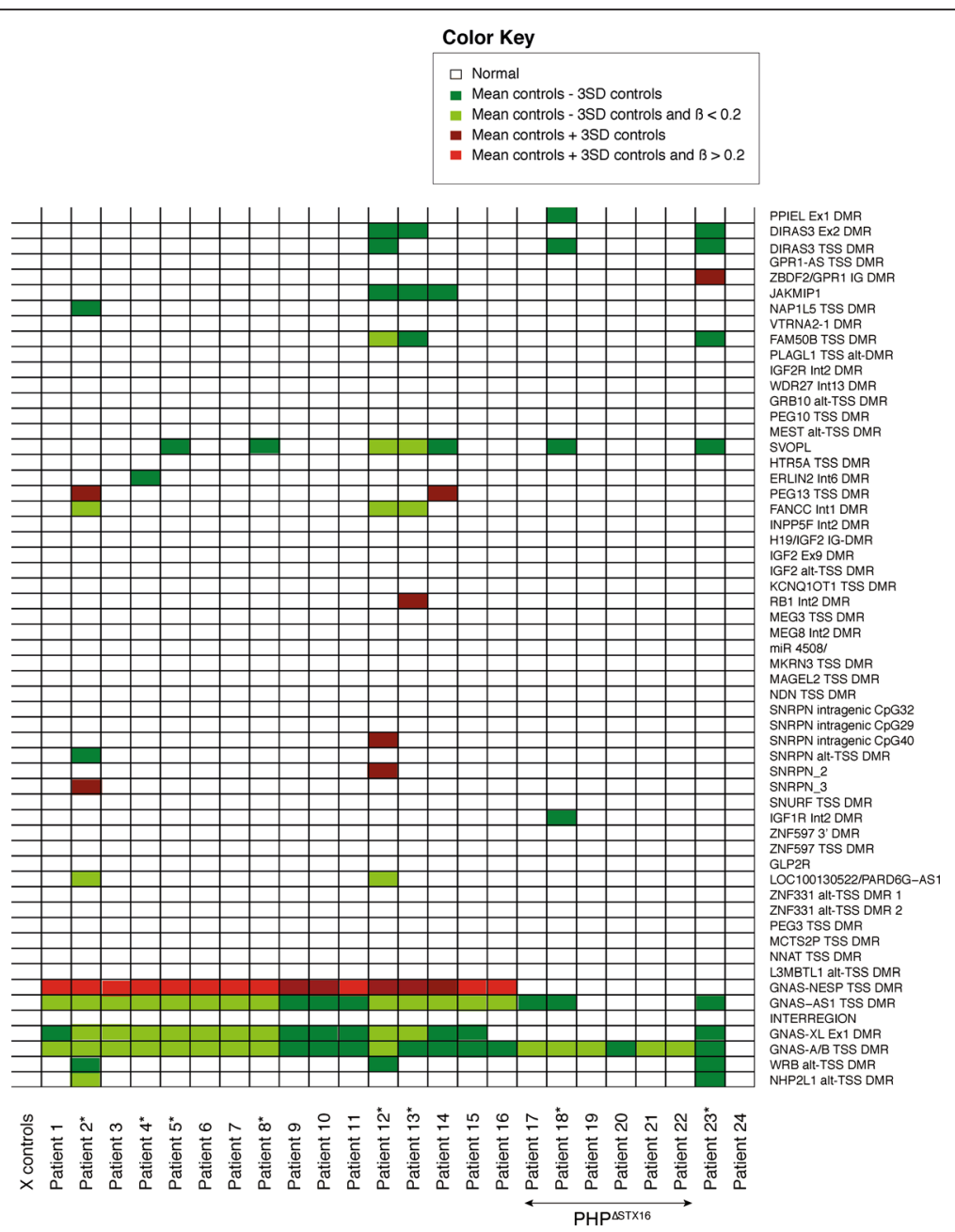

Fig. 5 Heatmap showing methylation of known imprinted genes in PHP patients. Methylation values of the genes are extracted from data obtained with the HM450k. Dark green and red respresent $-3 S D$ and $+3 S D$, respectively, of the mean as determined in 20 controls. Lighter green and red represent additional cut-offs for absolute methylation $<0.20$ and $>0.80$. Patients* have multi-locus methylation abnormalities. PHP ${ }^{\Delta S T X 16}$ patients with underlying STX16 deletion

improve our limited knowledge about imprinting disorders (IDs). There has been a revolution in DNA methylation analysis technology over the past decade as genome-wide and entire methylomes can be characterized at single-base-pair resolution [27]. Their implementation to study IDs has only just started. A custom Illumina GoldenGate array targeting 27 imprinted DMRs has been used to study 65 patients with an ID (that include Beckwith-Wiedemann syndrome (BWS), transient neonatal diabetes mellitus (TNDM), PHP1B, SilverRussell syndrome (SRS), Angelman syndrome (AS), and Prader-Willi syndrome (PWS)) and found multi-locus hypomethylation in patients with BWS, SRS, TNDM, and PHP1B, but not in AS and PWS [22]. Two studies have used the HM450k array to quantify genomewide DNA methylation for BWS and TNDM [28] or
SRS patients [29] and concluded that multiple and even novel imprinted genes were abnormally methylated in IDs.

Here, we have studied DNA methylation at about 485,000 CpGs genome-wide in 24 PHP patients with proven but different types of GNAS epimutations. The methylomes analyzed for 58 imprinted DMRs revealed in addition to the expected GNAS methylation defect, also a multi-locus methylation abnormality in 9 of the 24 patients for 19 other imprinted DMRs that were not previously known for these PHP cases. However, patients did not share a common pattern for these multi-locus methylation abnormalities, and there was a high variability in the degree of methylation difference and the number of amplicons per patient that were abnormally methylated. A validation study with the EpiTYPER for 


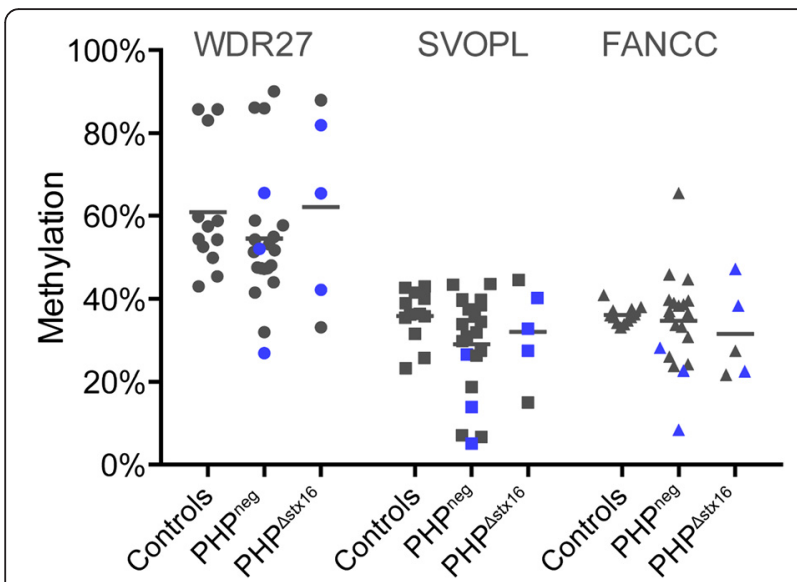

Fig. 6 Scatter plot of FANCC and SVOPL methylations in PHP patients and controls. Methylation values are obtained with the Sequenom EpiTYPER. Bar indicates the mean of the group. PHP patients are separated according to underlying STX16 deletion. Patients that were investigated with the HM450k are indicated in blue

the most common abnormally methylated DMRs FANCC and SVOPL indeed showed significant methylation differences in a replication cohort of PHP patients. The FANCC and SVOPL DMRs are not well studied, and further studies are needed to define the functional relevance of these methylation changes. Though larger cohorts are needed to be conclusive, based on results from both the HM450k and the EpiTYPER study, it seems that $\mathrm{PHP}^{\text {neg }}$ patients are more sensitive to these multi-locus defects compared to $\mathrm{PHP}^{\Delta \mathrm{stx} 16}$ patients. Other studies that have used targeted approaches with a limited number of known imprinted DMRs have also reported multi-locus defects in PHP patients. PerezNanclares et al. reported the first two PHP patients with multi-locus hypomethylation; one patient at PEG1/ $M E S T$ and the other at GTL2 [24]. Court et al. identified five PHP patients with hypomethylation at other imprinted loci: PEG1/MEST, MCTS2, IGF2R, ZNF331, L3MTBL1, and MEG3 [22]. Maupetit-Méhouas et al. studied a larger cohort of 63 PHP patients for the methylation pattern at eight imprinted loci and found multi-locus imprinting defects for 4 PHP patients at PEG1/MEST, L3MBTL1, and DLK1/GTL2 [23]. Though in our PHP cohort we observe a higher rate of multilocus methylation defects ( $38 \%$ of all patients), this is probably due to the fact that more DMRs were studied, but remarkably, most of these previously reported abnormal DMRs are also abnormal in our study. Docherty et al. identified novel candidate imprinted genes in patients with imprinting disorders and multi-locus methylation defects [28]; we identified hypomethylation of three candidate regions (PPIEL:Ex1-DMR, WRB:alt-TSSDMR, and NHP2L1:alt-TSS-DMR) from this study in our patients with PHP1B. The clinical relevance of these regions has not yet been determined. It is possible that these genes are common in most imprinting disorders. Interestingly, we identified two patients with methylation changes in the SNRPN DMRs. These two patients also have hypomethylation of the novel candidate DMR LOC100130522/PARD6G-AS1 [28]. Methylation changes in SNRPN DMRs are associated with PWS and AS, but these patients are very infrequent and moreover loss of methylation of the SNRPN DMR is rarely described in other imprinting disorders with multi-locus methylation disturbances. It has only been observed in two patients with SRS [30], two patients with BWS [22, 28], and one patient with TNDM [28]. In accordance with previous studies, our PHP patients with multi-locus methylation defects do not show evidence for phenotype differences with patients having only a GNAS methylation defect. Further studies including biochemical analyses are necessary to confirm these findings. The patient with the highest number of genes presenting with a methylation defect has severe hypertension with organ damage but a link with the methylation defect is not obvious. The other patients with multi-locus methylation defects have no additional clinical or molecular features. We identified five patients with simultaneous hypo- and hypermethylation. This finding is consistent with the report of Maupetit-Méhouas [23]. The epidominance hypothesis suggests that the phenotype is determined by the most strongly affected imprinted locus [23]. Patients with hypomethylation of multiple imprinted loci have been associated with increased frequency of developmental delay and congenital anomalies [31], but based on our current study, we have no evidence for a clinical impact. Of particular interest is the fact that methylation disturbances at multiple imprinted loci are now described in a subset of patients affected with different IDs, and this could point to a common pathogenic mechanism for IDs [32].

According to the original classification for PHP, we have included PHP1B patients with A/B only and partial or full broad methylation defects and PHP1A patients with a broad partial methylation defect. During the last years, it became clear for reasons specified in the introduction that this classification is no longer accurate. All CpGs from the genome-wide data within the GNAS cluster were analyzed using unsupervised hierarchical cluster analysis. This analysis divided our patients in two groups seemingly only dependent on having the STX16 deletion or not (referred to as $\mathrm{PHP}^{\Delta \mathrm{stx} 16}$ and $\mathrm{PHP}^{\text {neg }}$ ). The studied intervals cover the 4 DMRs in GNAS but these loci are much larger compared to the amplicons that were previously studied by the different labs that have used MS-MLPA, Sequenom EpiTYPER or pyrosequencing (for details see Fig. 1). PHP1B patients 23 and 24 that were diagnosed by MS-MLPA and 
pyrosequencing having a partial broad GNAS methylation defect are now clustered more closer to the control group. The most intriguing finding is however the discovery of a novel previously not described DMR (named GNAS-AS2) in the GNAS cluster that was found hypomethylated for both $\mathrm{PHP}^{\Delta \mathrm{stx} 16}$ and $\mathrm{PHP}^{\text {neg }}$ patients. GNAS-AS2 is located within the large GNAS-AS1 region and is actually located in the promoter region of the $X L$ exon 1 (Additional file 2: Figure S1) but is separated from $X L$ by a hypermethylated region. Many studies have described that PHP1B patients with STX16 deletion only have hypomethylation of the $A / B$ DMR while the DMRs $X L, G N A S-A S 1$, and NESP are normal [10-13]. This is also the case for the present HM450k data except for the CpGs that are located in the GNAS-AS2 region. GNAS-AS2 hypomethylation for both $\mathrm{PHP}^{\Delta \text { stx16 }}$ and $\mathrm{PHP}^{\text {neg }}$ patients was validated with the EpiTYPER. Only $1 / 24$ ( 1 with partial PHP1B-broad) and 4/ 42 PHP (3 with PHP1B-A/B only and 1 with partial PHP1B-broad) patients had a normal GNAS-AS2 methylation based on HM450k and EpiTYPER data, respectively. Methylation defects affecting the maternal $A / B$ DMR lead to the loss of Gs $\alpha$ expression in renal proximal tubules and renal PTH resistance. It is however not clear at this moment whether hypomethylation of the GNAS-AS2 DMR has a phenotype impact or whether methylation studies of this region can be useful for a better characterization of PHP patients. Further experiments are also needed to determine if loss of methylation of DMR GNAS-AS2 influences the expression of GNAS-XL.

\section{Conclusions}

We here present the first genome-wide methylation study in different types of PHP. It seems that only the presence of the STX16 deletion is of significant importance to divide PHP in two groups of patients with significantly different methylation profiles. Methylation changes in other imprinted DMRs seem to be enriched in especially PHP $^{\text {neg }}$ patients. We identified a novel GNAS-AS2 DMR in the GNAS locus that is hypomethylated in all PHP patients, independently from STX16 deletion. Further studies must be undertaken to unravel the function and clinical impact of methylation changes in the GNAS-AS2 region that in addition to the $A / B$ DMR can also be hypomethylation in PHP patients with STX16 deletion.

\section{Methods}

\section{Patient samples}

A total of 61 PHP patients were enrolled by 5 different endocrinology centers from the European PHP Consortium. The molecular diagnosis for PHP was performed by each center using the methylation detection assay for the GNAS DMRs as previously described with methodologies and the location of the studied amplicons in Fig. 1 and Table 1 [21]. All PHP patients included in this study have PTH resistance and were labeled with PHP1A if an obvious AHO phenotype was present (only brachydactyly and subcutaneous ossifications were noticed, Table 1). Based on the genetic screening for STX16 deletions and GNAS methylation screening, laboratories were asked to label the patients with the following: (i) STX16 deletion present or not, (ii) AB-only or broad methylation defect, and (iii) full or partial methylation defect [21]. This resulted in the use of the following labels (Table 1): PHP1B-A/B only, full PHP1B-broad, partial PHP1B-broad and partial PHP1A-broad. These terms are based on the original classification used for patients with PHP type I.

Table 1 presents the patients for the genome-wide study and Additional file 1: Table S2 the patients for the replication studies. The order of the patients in Table 1 was determined by unsupervised cluster analysis of genome-wide data for only the GNAS locus. The total number of PHP patients that were enrolled for the HM450k array and Sequenom studies are specified in Additional file 1: Table S2. Shortly, the genomewide DNA methylation study was performed for 24 PHP patients and 20 age- and gender-matched healthy controls (Table 1). Validation of NESP-AS2 was performed for 42 PHP patients and 20 controls (Additional file 1: Table S3). Validation of the methylation levels of FANCC, SVOPL, and WDR27 was performed in 26 PHP patients and 12 controls (Additional file 1: Table S6).

Informed consent for methylation and genetic studies was obtained from all participants and/or their legal representatives after approval of these studies by local Ethical Committees.

\section{Genome-wide methylation profiling using the HM450k array}

Genomic DNA was extracted from leukocytes using standard techniques and bisulfite converted using the EZ DNA methylation kit (Zymo Research, Irvine CA) as previously described [33]. The array was performed by the Genome Centre (Bart's and the London School of Medicine and Dentistry, Londen, UK) on the HumanMethylation 450K BeadChip (Illumina) using manufacturer's reagents and protocols. An identical control sample was assigned to each batch and samples were distributed randomly to control for batch effects. The correlation for the internal quality control was high (>0.99). The methylation level ( $\beta$ value) was calculated using the Methylation Module of BeadStudio software. 


\section{HM450k data filtering and genome-wide analysis}

After normalization of the data using GenomeStudio software, data were analyzed using a pipeline developed within the $\mathrm{R}$ statistical analysis environment (http://www.r-project.org, Bioconductor, Seattle, USA). Before analyzing the data, we excluded possible sources of technical bias. Probes with a high detection value $(p>0.01)$ in more than $10 \%$ of the samples (644 probes) and probes containing any missing values $(13,194$ probes) were removed, as well as nonCpG and gender-matched probes. Finally, we excluded probes as they contained SNPs present in $>1 \%$ of the population and leukocyte-specific probes [34, 35]. In total, we analyzed 355,105 probes for all DNA samples (73\% of probes). No statistical batch control was required as all the cases and controls had been processed in the same time, and the correlation for the quality control was high $(>0.99)$. The mean methylation and standard deviation were determined for the control population and individual samples. We did not detect any differentially methylated DMRs between the 20 control samples. DNA samples from imprinting syndrome patients were considered epimutated if the methylation value for an imprinted DMR differed was outside 3 standard deviations from the 20 control samples. Cluster analysis was performed within the $\mathrm{R}$ statistical analysis environment.

\section{HM450k data filtering for analysis of imprinted genes}

We analyzed 58 imprinted DMRs that also included the DMRs of the GNAS cluster (Additional file 1: Table S4). The list was based on the known imprinted DMRs from the consensus list from the European Network of Human Congenital Imprinting Disorders (http:// www.imprinting-disorders.eu). In addition, we included novel human imprinted DMRs from recent research that combined whole-genome bisulfite sequencing with HM450k to generate methylation profiles [36] as well as four novel candidate DMRs (GLP2R, JAKMIP1, LOC100130522/PARD6G-AS1, SVOPL) from research using genome-wide methylation profiling with HM450k in patients with multi-locus methylation defects [28]. These regions have not yet been further characterized to determine SNP versus parental-origin methylation and tissue profile. Some DMRs of the list are secondary DMRs (i.e., DIRAS3:Ex2-DMR, all the SNRPN DMRs).

\section{Validation of NESP-AS2 and FANCC, SVOPL, WDR27 methylation using the Sequenom EpiTYPER}

Leukocyte DNA $(1 \mu \mathrm{g})$ was subjected to bisulfite treatment using the MethylDetector ${ }^{\mathrm{mI}}$ bisulfite modification kit (Active Motif, Carlsbad CA, USA) as we described [21, 33, 36-38]. The Sequenom MassARRAY (Sequenom, San Diego, CA, USA) was used for quantitative
DNA methylation analysis of the CpGs within the amplicons of FANCC, SVOPL, and WDR27 using conditions described. Long cycling incubation was applied to further optimize the conversion reaction [39]. Primers were designed using the Sequenom EpiDesigner BETA software (www.epidesigner.com), taking into account amplicon coverage, number of $\mathrm{CpGs}$, fragment size and number of nucleotide repeats in the primer sequence (Additional file 1: Table S7). PCR steps were performed in triplicate for each DNA sample and a standard deviation between replicates was mostly $<10 \%$. When triplicate measurements had a SD $>10 \%$ or when only one of the triplicates was available, data for that sample were excluded. The mean of three values was used for further analyses. The EpiTYPER analysis method reports CpG methylation values as percentage. Statistical analyses to quantify DNA methylation differences were performed using the Prism 6 software (GraphPad Software Inc., San Diego). DNA samples from PHP patients were considered epimutated if the methylation value for an imprinted DMR was outside two and three standard deviations determined from the control samples. A twotailed $T$ test was used to assess differences in mean DNA methylation levels between cohorts for the overall amplicon considered as methylation average and for each CpG unit within this amplicon separately.

\section{GNAS molecular analysis}

Genomic DNA was isolated from leukocytes. Genotype for the GNAS exon 5 SNP (rs7121) at codon 131 was determined by PCR with the forward primer $5^{\prime}$ ttggtagcgccctcccaggc- $3^{\prime}$ and the reverse primer $5^{\prime}$ catgttcctatatggacactg- $3^{\prime}$. After denaturation at $95{ }^{\circ} \mathrm{C}$, 40 cycles of DNA amplification were performed using Taq PCR Mastermix at $95{ }^{\circ} \mathrm{C}$ for $30 \mathrm{~s}, 58{ }^{\circ} \mathrm{C}$ for $60 \mathrm{~s}$, and $72{ }^{\circ} \mathrm{C}$ for $60 \mathrm{~s}$. The PCR products were digested using the restriction enzyme FokI and analyzed on a $2 \%$ agarose gel. Only samples heterozygous at FokI polymorphism were selected. One patient and one control were heterozygous. Total RNA was extracted from platelets using TRIzol (Invitrogen) reagent, according to the manufacturer's protocol. The GNAS gene was amplified from platelet RNA to check expression of $X L$.

\section{Additional files}

Additional file 1: This contains additional files for Tables S1-S7.

Additional file 2: Detailed schematic representation of the human GNAS-AS region.

\section{Abbreviations}

A/B: exon A/B = GNAS-A/B:TSS DMR; AHO: albright hereditary Osteodystrophy; AS1: GNAS antisense = GNAS-AS1:TSS DMR; BWS: Beckwith-Wiedemann syndrome; DMRs: differentially methylated regions;

HM450k: HumanMethylation 450K BeadChip; NESP: neuroendocrine secretory 
protein 55 = GNAS-NESP:TSS DMR; PHP: pseudohypoparathyroidism; PPHP: pseudopseudohypoparathyroidism; PTH: parathyroid hormone; SRS: Silver-Russell syndrome; TNDM: transient neonatal diabetes mellitus; TSH: thyroid-stimulating hormone; XL: extra-large stimulatory $\mathrm{G}$ protein = GNAS-XL:EX1 DMR

\section{Competing interests}

The authors declare that they have no competing interests.

\section{Authors' contributions}

AR carried out the molecular genetic studies, analyzed the data, and drafted the manuscript. AR and AT developed the pipeline within the $R$ statistical analysis environment. BI, FE, IG, AL, GM, GPN, ST, CVG, AND BD performed the initial methylation studies and provided patient samples for the HM450k and the validation studies. KF and DM designed the research and helped to write the manuscript. All authors read and approved the final manuscript.

\section{Acknowledgements}

For this work, AR received a Short-Term Scientific Mission grant (within the framework of the COST-BM1209 project: European Network of Congenital Imprinting Disorders) to visit the lab of Dr. D Monk in the laboratory of Genomic Imprinting and Cancer at IDIBELL in Barcelona, Spain. This work was supported by the Euro-PseudoHypoParathyroidism network (EuroPHP) grant from the European Society for Pediatric Endocrinology Research Unit and by the Fund for Scientific Research-Flanders (FWO-Vlaanderen, Belgium) [G.0490.10 N and G.0A23.14 N]. All members of the EuroPHP are members of the EUCID.net (COST action BM1208 on imprinting disorders; http: //www.imprinting-disorders.eu).

\section{Members of the European Network of Human Congenital Imprinting Disorders}

Belgium (Kathleen Freson, Benedetta Izzi, Anne Rochtus, Department of Cardiovascular Sciences, Center for Molecular and Vascular Biology, University of Leuven), France (Agnes Linglart, Caroline Silve, Stephanie MaupetitMehouas and Virginie Grybek, INSERM U986, Hôpital Bicêtre, Le Kremlin Bicêtre, Paris; and Anne Barlier, Laboratory of Biochemistry and Molecular Biology, Conception Hospital, Aix-Marseille University, Marseille), Germany (Olaf Hiort, Susanne Thiele, Bettina Brix and Ralf Werner, Division of Experimental Paediatric Endocrinology and Diabetes, Department of Paediatrics, University of Luebeck), Italy (Giovanna Mantovani, Francesca M Elli, Fondazione IRCCS Ca' Granda Ospedale Maggiore Policlinico, Endocrinology and Diabetology Unit, Department of Clinical Sciences and Community Health, University of Milan, Milan; Luisa de Sanctis, University of Turin, Turin), Spain (Guiomar Perez de Nanclares, Intza Garin and Arrate Pereda, Molecular (Epi)Genetics Laboratory, BioAraba National Health Institute, Hospital Universitario Araba-Txagorritxu, Vitoria-Gasteiz; and Beatriz Lecumberri, Endocrinology Service, Hospital Universitario La Paz, Madrid), Turkey (Serap Turan, Marmara University School of Medicine Hospital, Istanbul) and United Kingdom (Deborah JG Mackay, Faculty of Medicine, University of Southampton, Southampton).

\section{Author details}

'Department of Cardiovascular Sciences, Center for Molecular and Vascular Biology, University of Leuven, Campus Gasthuisberg, O\&N1, Herestraat 49, Box 911, 3000 Leuven, Belgium. ²Department of Pediatrics, University Hospitals Leuven, 3000 Leuven, Belgium. '²aboratory of Genomic Imprinting and Cancer, IDIBELL, 08908 Barcelona, Spain. ${ }^{4}$ Fondazione IRCCS Ca' Granda Ospedale Maggiore Policlinico, Endocrinology and Diabetology Unit, Department of Clinical Sciences and Community Health, University of Milan, 20122, Milan, Italy. ${ }^{5}$ Molecular (Epi)Genetics Laboratory, BioAraba National Health Institute, Hospital Universitario Araba-Txagorritxu, 01009 Vitoria-Gasteiz, Spain. ${ }^{6}$ Department of Pediatric Endocrinology and Diabetology for Children, APHP, Bicêtre Paris Sud, 94275 Le Kremlin Bicêtre, France. ${ }^{7}$ Reference Center for Rare Disorders of the Mineral Metabolism and Plateforme d'Expertise Paris Sud, APHP, 94275 Le Kremlin Bicêtre, France. ${ }^{8}$ Division of Experimental Paediatric Endocrinology and Diabetes, Department of Paediatrics, University of Luebeck, 23560 Luebeck, Germany. ${ }^{9}$ Department of Clinical and Experimental Endocrinology, University of Leuven, 3000 Leuven, Belgium.
Received: 15 December 2015 Accepted: 17 January 2016 Published online: 26 January 2016

\section{References}

1. Peters $\mathrm{J}$. The role of genomic imprinting in biology and disease: an expanding view. Nat Rev Genet. 2014;15(8):517-30. doi:10.1038/nrg3766.

2. Levine MA. An update on the clinical and molecular characteristics of pseudohypoparathyroidism. Curr Opin Endocrinol Diabetes Obes. 2012;19(6): 443-51. doi:10.1097/MED.0b013e32835a255c.

3. Bastepe M. Genetics and epigenetics of parathyroid hormone resistance. Endocr Dev. 2013;24:11-24. doi:10.1159/000342494.

4. Patten JL, Johns DR, Valle D, Eil C, Gruppuso PA, Steele G, et al. Mutation in the gene encoding the stimulatory $\mathrm{G}$ protein of adenylate cyclase in Albright's hereditary osteodystrophy. N Engl J Med. 1990;322(20):1412-9. doi:10.1056/NEJM199005173222002.

5. Davies SJ, Hughes HE. Imprinting in Albright's hereditary osteodystrophy. J Med Genet. 1993;30(2):101-3.

6. Mantovani G. Clinical review: pseudohypoparathyroidism: diagnosis and treatment. J Clin Endocrinol Metab. 2011;96(10):3020-30. doi:10.1210/jc. 2011-1048.

7. de Nanclares GP, Fernandez-Rebollo E, Santin I, Garcia-Cuartero B, Gaztambide S, Menendez E, et al. Epigenetic defects of GNAS in patients with pseudohypoparathyroidism and mild features of Albright's hereditary osteodystrophy. J Clin Endocrinol Metab. 2007;92(6):2370-3. doi:10.1210/jc. 2006-2287.

8. Molinaro A, Tiosano D, Takatani R, Chrysis D, Russell W, Koscielniak N, et al. TSH elevations as the first laboratory evidence for pseudohypoparathyroidism type Ib (PHP-|b). J Bone Miner Res. 2015;30(5): 906-12. doi:10.1002/jbmr.2408.

9. Romanet P, Osei L, Netchine I, Pertuit M, Enjalbert A, Reynaud R, et al. Case report of GNAS epigenetic defect revealed by a congenital hypothyroidism. Pediatrics. 2015;135(4):e1079-83. doi:10.1542/peds.2014-2806.

10. Bastepe M, Pincus JE, Sugimoto T, Tojo K, Kanatani M, Azuma Y, et al. Positional dissociation between the genetic mutation responsible for pseudohypoparathyroidism type $\mathrm{lb}$ and the associated methylation defect at exon A/B: evidence for a long-range regulatory element within the imprinted GNAS1 locus. Hum Mol Genet. 2001;10(12):1231-41.

11. Bastepe M, Frohlich LF, Hendy GN, Indridason OS, Josse RG, Koshiyama H, et al. Autosomal dominant pseudohypoparathyroidism type $\mathrm{lb}$ is associated with a heterozygous microdeletion that likely disrupts a putative imprinting control element of GNAS. J Clin Invest. 2003;112(8): 1255-63. doi:10.1172/JCl19159.

12. Linglart A, Gensure RC, Olney RC, Juppner $H$, Bastepe M. A novel STX16 deletion in autosomal dominant pseudohypoparathyroidism type Ib redefines the boundaries of a cis-acting imprinting control element of GNAS. Am J Hum Genet. 2005;76(5):804-14. doi:10.1086/429932.

13. Elli FM, de Sanctis L, Peverelli E, Bordogna P, Pivetta B, Miolo G, et al. Autosomal dominant pseudohypoparathyroidism type lb: a novel inherited deletion ablating STX16 causes loss of imprinting at the A/B DMR. J Clin Endocrinol Metab. 2014;99(4):E724-8. doi:10.1210/jc.2013-3704.

14. Richard N, Abeguile G, Coudray N, Mittre H, Gruchy N, Andrieux J, et al. A new deletion ablating NESP55 causes loss of maternal imprint of A/B GNAS and autosomal dominant pseudohypoparathyroidism type Ib. J Clin Endocrinol Metab. 2012;97(5):E863-7. doi:10.1210/jc.2011-2804.

15. Unluturk U, Harmanci A, Babaoglu M, Yasar U, Varli K, Bastepe M, et al. Molecular diagnosis and clinical characterization of pseudohypoparathyroidism type-lb in a patient with mild Albright's hereditary osteodystrophy-like features, epileptic seizures, and defective renal handling of uric acid. Am J Med Sci. 2008;336(1):84-90. doi:10.1097/MAJ.0b013e31815b218f.

16. Mantovani G, de Sanctis L, Barbieri AM, Elli FM, Bollati V, Vaira V, et al. Pseudohypoparathyroidism and GNAS epigenetic defects: clinical evaluation of albright hereditary osteodystrophy and molecular analysis in 40 patients. J Clin Endocrinol Metab. 2010;95(2):651-8. doi:10.1210/jc.2009-0176.

17. Mariot V, Maupetit-Mehouas S, Sinding C, Kottler ML, Linglart A. A maternal epimutation of GNAS leads to Albright osteodystrophy and parathyroid hormone resistance. J Clin Endocrinol Metab. 2008;93(3):661-5. doi:10.1210/jc.2007-0927.

18. Elli FM, de Sanctis L, Bollati V, Tarantini L, Filopanti M, Barbieri AM et al. Quantitative analysis of methylation defects and correlation with clinical characteristics in patients with pseudohypoparathyroidism type I and 
GNAS epigenetic alterations. J Clin Endocrinol Metab. 2013:jc20133086. doi:10.1210/jc.2013-3086.

19. Freson K, Izzi B, Labarque V, Van Helvoirt M, Thys C, Wittevrongel C, et al. GNAS defects identified by stimulatory $G$ protein alpha-subunit signalling studies in platelets. J Clin Endocrinol Metab. 2008;93(12):4851-9. doi:10.1210/jc.2008-0883.

20. Zazo C, Thiele S, Martin C, Fernandez-Rebollo E, Martinez-Indart L, Werner R, et al. Gsalpha activity is reduced in erythrocyte membranes of patients with psedohypoparathyroidism due to epigenetic alterations at the GNAS locus. J Bone Miner Res. 2011;26(8):1864-70. doi:10.1002/jbmr.369.

21. Garin I, Mantovani G, Aguirre U, Barlier A, Brix B, Elli FM, et al. European guidance for the molecular diagnosis of pseudohypoparathyroidism not caused by point genetic variants at GNAS: an EQA study. Eur J Hum Genet. 2015;23(4):438-44. doi:10.1038/ejhg.2014.127.

22. Court F, Martin-Trujillo A, Romanelli V, Garin I, Iglesias-Platas I, Salafsky I, et al. Genome-wide allelic methylation analysis reveals disease-specific susceptibility to multiple methylation defects in imprinting syndromes. Hum Mutat. 2013;34(4):595-602. doi:10.1002/humu.22276.

23. Maupetit-Mehouas S, Azzi S, Steunou V, Sakakini N, Silve C, Reynes C, et al. Simultaneous hyper- and hypomethylation at imprinted loci in a subset of patients with GNAS epimutations underlies a complex and different mechanism of multilocus methylation defect in pseudohypoparathyroidism type 1b. Hum Mutat. 2013;34(8):1172-80. doi:10.1002/humu.22352.

24. Perez-Nanclares G, Romanelli V, Mayo S, Garin I, Zazo C, Fernandez-Rebollo $E$, et al. Detection of hypomethylation syndrome among patients with epigenetic alterations at the GNAS locus. J Clin Endocrinol Metab. 2012; 97(6):E1060-7. doi:10.1210/jc.2012-1081.

25. Kobayashi H, Yanagisawa E, Sakashita A, Sugawara N, Kumakura S, Ogawa H, et al. Epigenetic and transcriptional features of the novel human imprinted IncRNA GPR1AS suggest it is a functional ortholog to mouse Zdbf2linc. Epigenetics. 2013;8(6):635-45. doi:10.4161/epi.24887.

26. Boycott KM, Vanstone MR, Bulman DE, MacKenzie AE. Rare-disease genetics in the era of next-generation sequencing: discovery to translation. Nat Rev Genet. 2013;14(10):681-91. doi:10.1038/nrg3555.

27. Laird PW. Principles and challenges of genomewide DNA methylation analysis. Nat Rev Genet. 2010;11(3):191-203. doi:10.1038/nrg2732.

28. Docherty LE, Rezwan FI, Poole RL, Jagoe H, Lake H, Lockett GA, et al. Genomewide DNA methylation analysis of patients with imprinting disorders identifies differentially methylated regions associated with novel candidate imprinted genes. J Med Genet. 2014;51(4):229-38. doi:10.1136/jmedgenet-2013-102116.

29. Prickett AR, Ishida M, Bohm S, Frost JM, Puszyk W, Abu-Amero S, et al. Genome-wide methylation analysis in Silver-Russell syndrome patients. Hum Genet. 2015;134(3):317-32. doi:10.1007/s00439-014-1526-1.

30. Azzi S, Rossignol S, Steunou V, Sas T, Thibaud N, Danton F, et al. Multilocus methylation analysis in a large cohort of 11p15-related foetal growth disorders (Russell Silver and Beckwith Wiedemann syndromes) reveals simultaneous loss of methylation at paternal and maternal imprinted loci. Hum Mol Genet. 2009;18(24):4724-33. doi:10.1093/hmg/ddp435.

31. Poole RL, Docherty LE, Al Sayegh A, Caliebe A, Turner C, Baple E, et al. Targeted methylation testing of a patient cohort broadens the epigenetic and clinical description of imprinting disorders. Am J Med Genet A. 2013; 161(9):2174-82. doi:10.1002/ajmg.a.36049.

32. Eggermann T, Elbracht M, Schroder C, Reutter $H$, Soellner L, Spengler $S$, et al. Congenital imprinting disorders: a novel mechanism linking seemingly unrelated disorders. J Pediatr. 2013;163(4):1202-7. doi:10.1016/j.jpeds.2013.05.017.

33. Rochtus A, Izzi B, Vangeel E, Louwette S, Wittevrongel C, Lambrechts D, et al. DNA methylation analysis of homeobox genes implicates HOXB7 hypomethylation as risk factor for neural tube defects. Epigenetics. 2015: 10(1):92-101. doi:10.1080/15592294.2014.998531.

34. Reinius LE, Acevedo N, Joerink M, Pershagen G, Dahlen SE, Greco D, et al. Differential DNA methylation in purified human blood cells: implications for cell lineage and studies on disease susceptibility. PLoS One. 2012;7(7), e41361. doi:10.1371/journal.pone.0041361.

35. Houseman EA, Accomando WP, Koestler DC, Christensen BC, Marsit CJ, Nelson $\mathrm{HH}$, et al. DNA methylation arrays as surrogate measures of cell mixture distribution. BMC bioinformatics. 2012;13:86. doi:10.1 186/1471-2105-13-86.

36. Court F, Tayama C, Romanelli V, Martin-Trujillo A, Iglesias-Platas I, Okamura $\mathrm{K}$, et al. Genome-wide parent-of-origin DNA methylation analysis reveals the intricacies of human imprinting and suggests a germline methylationindependent mechanism of establishment. Genome Res. 2014;24(4):554-69. doi:10.1101/gr.164913.113.
37. Izzi B, Decallonne B, Devriendt K, Bouillon R, Vanderschueren D, Levtchenko $\mathrm{E}$, et al. A new approach to imprinting mutation detection in GNAS by Sequenom EpiTYPER system. Clin Chim Acta. 2010;411(23-24): 2033-9. doi:10.1016/j.cca.2010.08.034.

38. Izzi B, Francois I, Labarque V, Thys C, Wittevrongel C, Devriendt K, et al. Methylation defect in imprinted genes detected in patients with an Albright's hereditary osteodystrophy like phenotype and platelet Gs hypofunction. PLoS One. 2012;7(6), e38579. doi:10.1371/journal.pone.0038579.

39. Izzi B, Binder AM, Michels KB. Pyrosequencing evaluation of widely available bisulfite conversion methods: considerations for application. Med Epigenet. 2014;2(1):28-36. doi:10.1159/000358882.

\section{Submit your next manuscript to BioMed Central and we will help you at every step:}

- We accept pre-submission inquiries

- Our selector tool helps you to find the most relevant journal

- We provide round the clock customer support

- Convenient online submission

- Thorough peer review

- Inclusion in PubMed and all major indexing services

- Maximum visibility for your research

Submit your manuscript at www.biomedcentral.com/submit
(O) BioMed Central 